\title{
Development of a Generic Anti-PEG Antibody Assay Using BioScale's Acoustic Membrane MicroParticle Technology
}

\author{
Huijin Dong, ${ }^{1,3}$ Johanna R. Mora, ${ }^{1}$ Catherine Brockus, ${ }^{1}$ Shannon D. Chilewski, ${ }^{1}$ Robert Dodge, ${ }^{1}$ \\ Colin Merrifield, ${ }^{2}$ W. Matthew Dickerson, ${ }^{2}$ and Binodh DeSilva ${ }^{1}$
}

Received 7 January 2015; accepted 8 June 2015; published online 3 July 2015

\begin{abstract}
Immunogenicity testing for PEGylated biotherapeutics should include methods to detect both anti-protein and anti-PEG antibodies (anti-PEG). Although some methods have been published for the detection of anti-PEG antibodies, the information is incomplete and, in some cases, reagents used (such as Tween-20) are known to interfere with detection. This rapid communication describes the use of BioScale's Acoustic Membrane MicroParticle (AMMP®) technology using the ViBE® Workstation to measure anti-PEG antibodies in human serum samples. Briefly, a sample spiked with monoclonal human $\mathrm{IgG}$ anti-PEG antibody is diluted in buffer and incubated with paramagnetic beads coated with linear chain $\mathrm{mPEG}$ to capture anti-PEG antibodies. The complex is then captured on an acoustic membrane coated with Protein A. The change in mass on the membrane caused by the binding of the complex to the membrane results in a signal proportional to the mass of anti-PEG antibodies. The data indicate that an assay with a sensitivity of less than $1000 \mathrm{ng} / \mathrm{mL}$ for $\mathrm{IgG}$ is achievable. This level of sensitivity is better than current published reports on IgG anti-PEG antibody detection.
\end{abstract}

KEY WORDS: acoustic membrane microparticle technology; anti-peg antibodies; emerging technology; immunogenicity assays; pegylated biotherapeutics.

\section{INTRODUCTION}

PEGylation is a well-documented modification used to increase therapeutic protein half-life. However, immune responses to the PEG itself have caused, in some cases, loss of product efficacy and adverse safety consequences, which highlights the importance of developing a strategy to monitor anti-PEG antibodies based on risk assessment (1). Also, the recently published FDA Guidance for Industry on Immunogenicity Assessment for Therapeutic Protein Products has recommended that for PEGylated therapeutic protein products, anti-drug antibody (ADA) assays should be able to detect both anti-protein antibodies and antibodies against the PEG moiety (2). This recommendation has proven to be a tall order, as developing and validating assays to detect antibodies against a PEG moiety is a major challenge. In a review paper by Schellekens et al. (3), the authors concluded that most, if not all, assays used for detecting anti-PEG antibodies are flawed due to the lack of specificity as well as poor characterization of positive controls $(3,4)$. Until recently,

\footnotetext{
${ }^{1}$ Bioanalytical Sciences Biologics, Bristol-Myers Squibb, Princeton, New Jersey 08543, USA.

${ }^{2}$ BioScale, Billerica, Massachusetts 01821, USA.

${ }^{3}$ To whom correspondence should be addressed. (e-mail: Huijin.dong@bms.com)
}

traditional bridge immunoassay format assays have been able to detect anti-PEG $\operatorname{IgM}$ antibodies but have struggled to detect IgG isotype antibodies with sufficient sensitivity in human matrix $(5,6)$, suggesting that the type of PEG and/or protein therapeutic may play a role. In addition to previously published comments (7), our own observations during assay development have noted that high levels of $\mathrm{IgG}$ in a sample make detection of low affinity anti-PEG IgG antibodies difficult in a plate-based or non-plate-based assay format using anti-human IgG detection reagents. A wellcharacterized antibody positive control and robust assay to detect anti-PEG IgG isotype will help to understand the mechanism of induced anti-PEG response following PEGylated therapeutic protein injection in human $(8,9)$. In this rapid communication, we report preliminary results for detecting IgG anti-PEG antibodies using an Acoustic Membrane MicroParticle (AMMP) platform.

The Acoustic Membrane MicroParticle platform is an emerging technology that utilizes a non-optical detection system to determine analyte concentration by measuring the change in the oscillating frequency of a piezoelectric membrane (10). This rapid communication describes a method in which human serum spiked with monoclonal chimeric IgG is diluted in buffer and incubated with paramagnetic beads coated with either PEGylated therapeutic protein or biotinylated PEG to capture anti-PEG antibodies. The complex is then detected by magnetically pulling all paramagnetic beads 
onto an acoustic membrane sensor coated with Protein A. Beads that are complexed with anti-PEG antibodies remain bound to the membrane sensor through the Protein A, following removal of the magnet. The change in mass on the membrane results in a signal proportional to the mass of anti-PEG antibodies.

Biotinylated PEG of various molecular weights can be coupled to streptavidin-coated paramagnetic beads, making this technology able to detect anti-PEG antibodies against a variety of PEG molecules. The work presented here focuses on the implementation of AMMP for the detection of antiPEG antibodies for immunogenicity assessment.

\section{MATERIALS AND METHODS}

\section{Materials}

\section{Commercial Reagents}

Biotin-PEG $20 \mathrm{kDa}$ and biotin-PEG $40 \mathrm{kDa}$ were purchased from Nanocs (www.nanocs.net, New York, NY). All biotinylated PEG molecules used in this study as well as in positive control characterization were linear chain molecules with a single biotin attached at one end and a methyl cap at the other end except for the PEG (40 kDa branched) attached to BMS drug A. The following buffers were purchased from Thermo Fisher Scientific, Waltham, MA: Blocker Casein in phosphate-buffered saline (PBS) with $1 \%$ $(w / v)$ casein (Hammarsten grade), $\mathrm{pH} 7.4$; Super Block buffer in PBS with proprietary protein, $\mathrm{pH} 7.4$; and Super Block buffer in Tris-buffered saline (TBS) with proprietary protein, $\mathrm{pH}$ 7.4. Normal human sera were purchased from Bioreclamation LLC, Westbury, NY. AMMP Type I Labeling Kit for Assay Discovery, AMMP Type II Labeling Kit for Assay Discovery, and Protein A Cartridges with ViBE Cartridge Regeneration Buffer were purchased from BioScale, Billerica, MA. Polypropylene plates (96-well) for inline incubations were purchased from BioScale.

\section{Proprietary Reagents}

Drug A is a BMS biotherapeutic with a $40 \mathrm{kDa} P E G$ attached to a $12 \mathrm{kDa}$ protein.

Custom-made monoclonal anti-PEG antibody (PEG.2): Briefly, mice were immunized at BMS with a panel of PEGylated BMS therapeutics and hybridomas were selected that showed binding to PEG coupled to unrelated protein to ensure anti-PEG reactivity. The variable region genes from antibody production cell line were sequenced, subcloned into human IgG1 Fc bearing constructs, stably expressed in $\mathrm{CHO}$ cell lines and purified. The binding of PEG.2 was analyzed on a Biacore T200 SPR instrument (GE Healthcare, Piscataway, NJ, USA) by immobilization of different MW biotin-mPEG onto a Neutravidin-coated sensor. PEG.2 showed the highest normalized binding response units (RU) to the $20 \mathrm{kDa} P E G$ compared to 5, 10, 30, and $40 \mathrm{kDa}$ PEG. Also, amine-PEGamine (aPEGa from Creative PEGWorks with a linear formula $\mathrm{NH}_{2}\left(\mathrm{CH}_{2}\right)_{2} \mathrm{O}\left(\mathrm{CH}_{2} \mathrm{CH}_{2} \mathrm{O}\right)_{n}\left(\mathrm{CH}_{2}\right)_{2} \mathrm{NH}_{2}$ and $\mathrm{MW}$ 20,000) was a stronger inhibitor than triethylene glycol monomethyl ether (mTEG from Sigma-Aldrich Corp. with linear formula $\mathrm{CH}_{3}\left(\mathrm{OCH}_{2} \mathrm{CH}_{2}\right)_{3}$ and $\mathrm{MW}$ 164.20) in the binding of PEG.2 to biotin-PEG on a plate suggesting that PEG.2 is a backbone unit-specific antibody (11).

\section{METHODS}

\section{Capture Reagent}

\section{Epoxy Bead Labeling}

PEGylated BMS drug A was directly conjugated to epoxy beads (BioScale's AMMP Type II Labeling Kit) per manufacturer's procedure.

\section{Streptavidin Bead Labeling}

An AMMP Type I Labeling Kit was used for binding of biotinylated PEG of 20 and $40 \mathrm{kDa}$ to paramagnetic streptavidin beads. The method for binding biotinylated PEG to the beads was modified slightly. Briefly, biotinylated PEG was diluted in PBS buffer and reacted with streptavidin beads. The manufacturer's recommendations were followed except that biotinylated bovine serum albumin (BSA) was not used to saturate open binding sites that were not specifically blocked with a biotinylated reagent.

\section{Protein A Detection With or Without Off-Line Bead Wash}

The ViBE platform, for this assay, uses Protein A on a piezoelectric membrane to capture the antibody complex. In the detection step, bound antibody complexes are separated from other matrix components present in the sample by magnetically attracting the beads to the membrane surface, but allowing beads without bound anti-PEG to fall away from the protein A-coated membrane as the magnet is then removed.

Experiments were designed to compare the assay with or without off-line wash, that is, homogenous $v s$. a nonhomogeneous assay. PEG.2 dilutions ranging from 0.250 to $4.0 \mu \mathrm{g} / \mathrm{mL}$ were prepared in PBS with $1 \%$ BSA $(w / v)$ and incubated with epoxy beads conjugated with drug A for $1 \mathrm{~h}$. The plate was then placed either on a BioTek washer equipped with a magnet block to allow aspiration of nonbinding components, or analyzed on the ViBE without the wash step. In both cases, a Protein A surface was used for detection.

\section{Buffer Selections}

Multiple buffers were tested for suitability, and three buffers (Blocker Casein in PBS, Super Block in PBS, and Super Block in TBS) were further tested for bead blocking and sample dilution. Conjugated type I beads were diluted in each buffer and blocked for $2 \mathrm{~h}$ at ambient temperature prior to use. Control samples were prepared by adding PEG.2 positive control to normal human serum pool at 0.625 to $40 \mu \mathrm{g} / \mathrm{mL}$ and diluted to a final concentration of $5 \%(v / v)$ in each of the buffers. Detection of the controls was evaluated using both sets of biotin-20 kDa PEG and biotin-40 kDa PEG type I beads (20 and $30 \mu \mathrm{g}$ PEG/mg beads) without off-line wash. Blocking and serum dilutions were paired for these comparisons. 
Assay Sensitivity and Reproducibility Using Optimized Assay Format

The optimized assay format using $10 \%$ serum $(v / v)$ is shown in Fig. 1. Control samples were prepared by adding PEG.2 positive control to normal human serum pool at 0.625 to $40 \mu \mathrm{g} / \mathrm{mL}$ and stored at $-70^{\circ} \mathrm{C}$ for $24 \mathrm{~h}$ prior to use. The spiked controls were thawed at room temperature and diluted tenfold in Blocker Casein in PBS. To perform the assay, Biotin-PEG $20 \mathrm{kDa}$ labeled beads were first diluted in Blocker Casein in PBS and incubated for $1.5 \mathrm{~h}$ at room temperature. Then, $80 \mu \mathrm{L}$ of each positive control diluted to $10 \%$ serum were combined with $40 \mu \mathrm{L}$ of blocked bead solution in a 96-well polypropylene plate and incubated for $1 \mathrm{~h}$ on the ViBE instrument integrated shaker. Once the incubation was complete, the online assay steps were initiated and data were collected by the ViBE software version 0.7.4.14126. The controls were run a total of 12 times over 2 days, one plate per day. Each plate contained six replicates. The mean response and intra- and inter-run \%CV were calculated.

\section{RESULTS}

\section{Assay Format Using Protein A Detection With or Without off-Line Bead Wash}

The assay using drug A on epoxy (type II) beads with Protein A cartridges had better sensitivity without using the off-line wash than when the off-line wash was performed. Accumulation values recorded by the ViBE are the measurement of the number of beads in the reaction. The data indicated that magnet-separated samples (pre-treatment step) had lower and less consistent bead accumulation counts than the samples without a magnetic separation pre-treatment step. Mean bead values for samples in 96 wells without a magnetic separation pretreatment step were more than twofold greater than for

\section{Functionalized Sensor with Protein A}

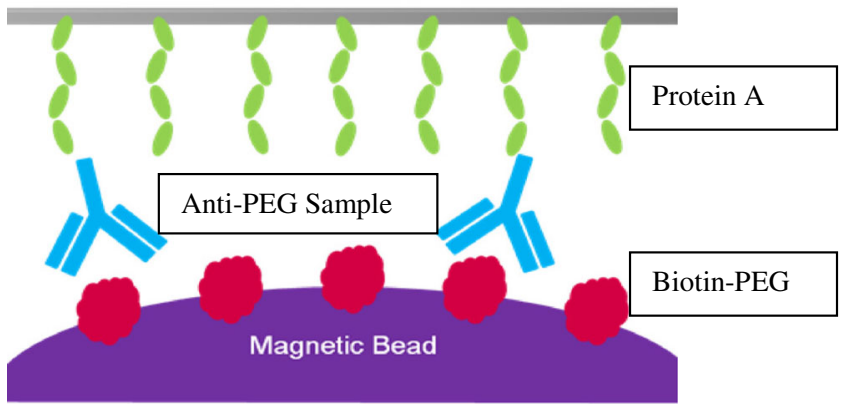

Fig 1. AMMP assay format: anti-PEG detection with biotin-PEG $20 \mathrm{kDa}$ immobilized on the magnetic bead and detection of complex on the cartridge coated with Protein A. Biotin-PEG is coupled onto streptavidin-coated paramagnetic beads. Anti-PEG in the human serum forms complexes on the coated beads under homogeneous conditions. When samples flow across the sensor cartridge, the bead complex is captured by Protein A bound to the sensor surface, a piezoelectric membrane. This change in mass on the sensor generates a signal proportional to the mass of bound anti-PEG magnetic separated reactions and bead values in 96 wells had a good precision $(\% \mathrm{CV} \leq 6.7)$. These data are summarized in Fig. 2.

\section{Buffer Selections for Blocking and Sample Dilution}

Using streptavidin (SA) coated type I beads and two loading concentrations of biotin-PEG $20 \mathrm{kDa}$, Blocker Casein in PBS had the lowest background signal and resulted in the best assay sensitivity as shown in Fig. 3. Differences between the 20 and $30 \mu \mathrm{g} / \mathrm{mg}$ loads indicated that the lower coating concentration was more effective at providing higher sensitivity $(<1000 \mathrm{ng} / \mathrm{mL})$ as well as a greater range of detection to up to $40 \mu \mathrm{g} / \mathrm{mL}$, the maximum level tested. Similar results were obtained using $40 \mathrm{kDa}$ biotin-PEG beads (not shown).

\section{Assay Sensitivity Using Optimized Format}

The sensitivity of the optimized assay was assessed in human serum using PEG.2 anti-PEG antibody as a positive control. Data were normalized to the negative control value for each dilution buffer on each plate. A normalized curve is shown in Fig. 4. Approximate sensitivity of the ViBE method using $10 \%$ human serum in Blocker Casein in PBS and $20 \mathrm{kDa}$ PEG/20 $\mu \mathrm{g} / \mathrm{mg}$ beads with the PEG.2 positive control is $800 \mathrm{ng} / \mathrm{mL}$ based on lowest control concentration above a cut point. The cut point was calculated according to the formula of average $+1.645 \times \mathrm{SD}_{\text {Mean }}$, where the average of 0.14 and standard deviation of 12 replicates of unspiked human serum pool were used. Assay reproducibility was evaluated using the same runs as for sensitivity, one plate per day for each of 2 days. Each plate consisted of six replicates of the titration curve prepared as tenfold dilutions in Blocker Casein in PBS against $20 \mathrm{kDa}$ PEG $/ 20 \mu \mathrm{g} / \mathrm{mg}$ beads. Interplate and intra-plate variability for the replicates were indicated by $\leq 6.7 \% \mathrm{CV}$ and $1.8 \% \mathrm{CV}$, respectively, as shown in Table I.

\section{DISCUSSION}

Reports $(12,13)$ of both pre-existing and therapeutically induced anti-PEG antibodies highlight the importance of developing a reliable assay for their detection. The development of an assay for the detection of antiPEG antibodies has proven to be challenging. Some assays have been developed to measure $\operatorname{IgM}$ response, but the measurement of $\mathrm{IgGs}$ is more complicated because of the high levels of other human IgGs interfering when using an anti-human $\mathrm{Fc}$ in the assay. Also, we hypothesize that anti-PEG IgGs may be less successful at bridging two distinctly labeled PEG molecule instead of both antibody "arms" binding two units within the same molecule.

Using PEG.2 as a positive control antibody, a generic Acoustic Membrane Micro Particle assay using the ViBE instrument platform for the detection of antiPEG antibodies in human serum has been successfully developed. It is possible that this assay on the ViBE can overcome issues observed in other assays regarding high 
a

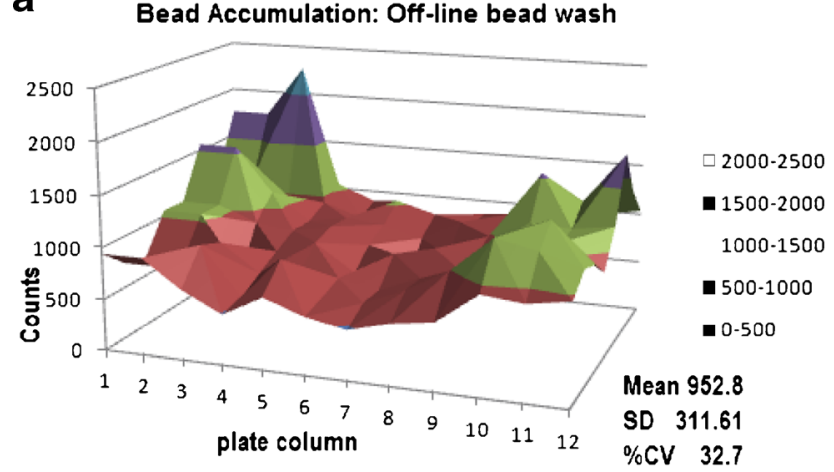

b

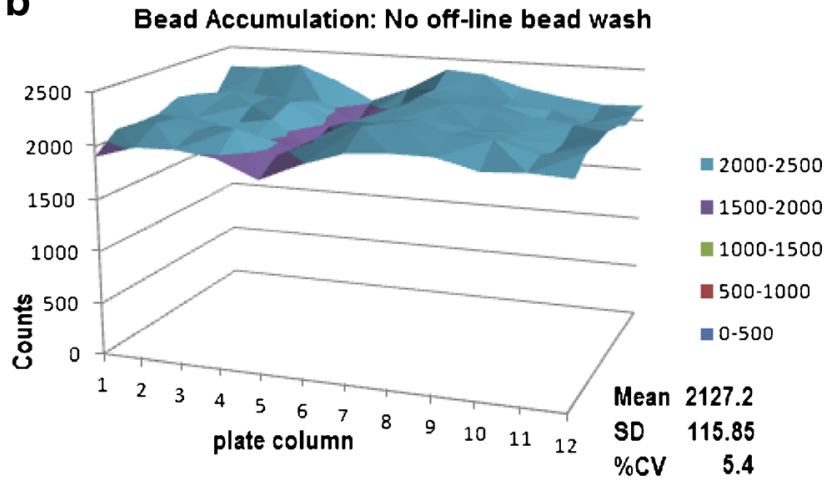

Fig 2. Comparative 3-D graphs of bead accumulation values across assay plates using a an off-line bead wash and $\mathbf{b}$ without off-line bead wash prior to loading onto the ViBE analyzer. The bead accumulation value is the number of beads captured at sensor surface when the magnet is engaged. Magnetic separation was achieved by using a BioTek microwell plate washer fitted with a magnetic plate to draw down beads suspended in solution, followed by aspiration of supernatant. Bead accumulation without off-line bead wash was superior to results obtained with an off-line bead wash because some of the beads were lost during the wash. Assay format without off-line bead wash was selected for the final method development

background signal due to non-specific binding thanks to the following factors:

a) In the ViBE platform, bound antibody complexes are separated from other matrix components present in the sample by attracting beads to the membrane surface magnetically, but allowing unbound beads to fall away from the membrane as the magnet is then removed. Other proteins in sample may bind to the Protein A surface, but if they are not complexed to magnetic

\section{Three Buffer Comparison: $20 \mu \mathrm{g}$ vs. $30 \mu \mathrm{g}$ Type I coated Beads}

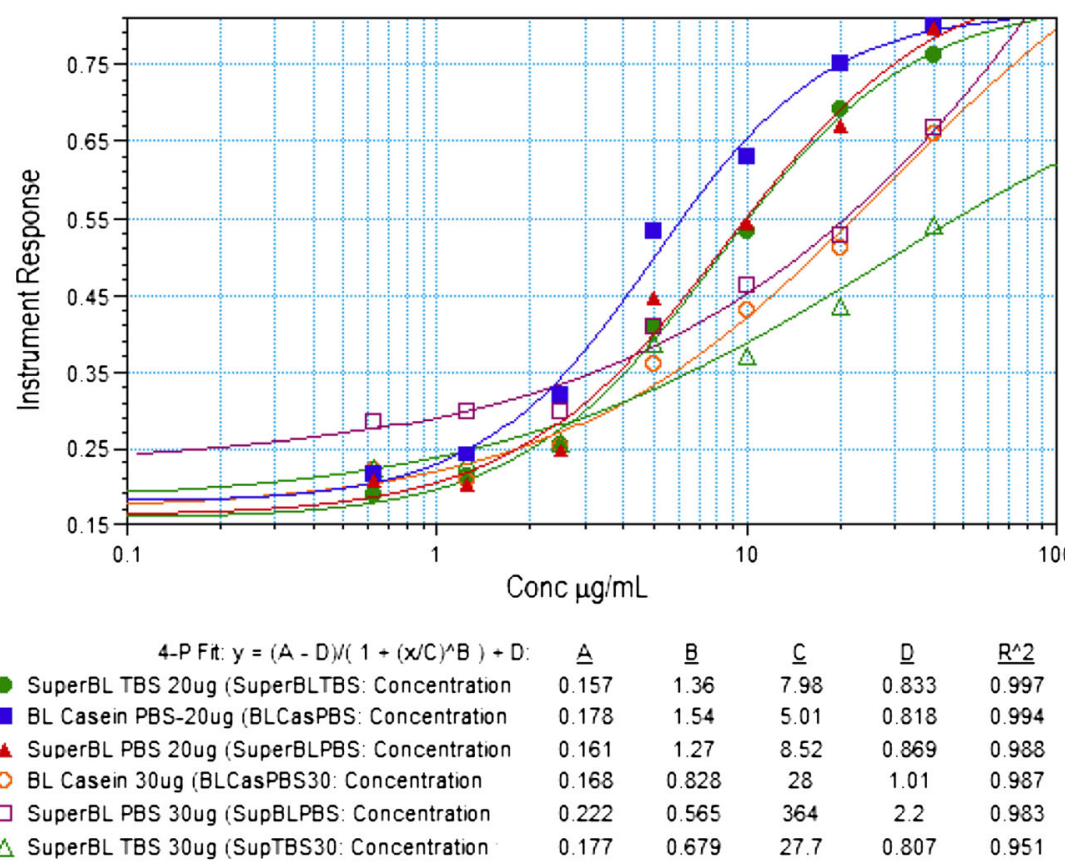

Weighting: Foxed

Fig 3. Buffer effect on assay background and sensitivity. Positive control curves were prepared in buffer containing $5 \%(v / v)$ human serum and assay was performed with biotinPEG $20 \mathrm{kDa}$ using type I streptavidin beads coated with 20 or $30 \mu \mathrm{g}$ biotin-PEG. Reduced slopes, sensitivity, and higher background values were found using $30 \mu \mathrm{g}$ coated beads, regardless of buffer used. The combination including Blocker Casein in PBS with $20 \mu \mathrm{g}$ coated beads was selected for further testing 


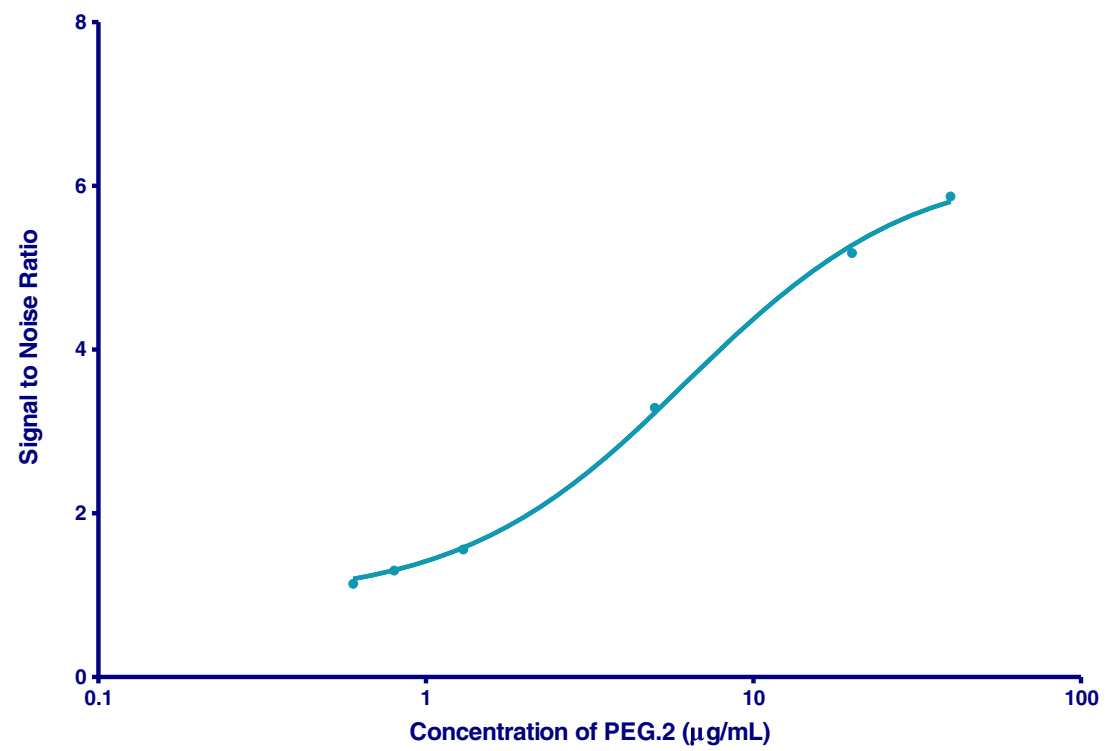

Fig 4. Dose-response curve (4PL regression using GraphPad) of the immunogenicity ViBE assay for the detection of anti-PEG antibodies. The observed sensitivity based on the 95th percentile confidence interval was $800 \mathrm{ng} / \mathrm{mL}$. Calibrators were prepared by spiking PEG.2 positive control into the normal human serum at 0.625 to $40 \mu \mathrm{g} / \mathrm{mL}$ and normal human serum was used as negative control. Calibrators and negative control were diluted tenfold in Blocker Casein in PBS. Biotin-PEG $20 \mathrm{kDa}$ labeled beads at $20 \mu \mathrm{g} / \mathrm{mg}$ were reacted in a homogeneous mixture with $80 \mu \mathrm{L}$ of each spiked control in $10 \%$ serum in a 96well polypropylene plate and incubated for $1 \mathrm{~h}$ on the ViBE instrument integrated shaker. Signal-to-noise ratio=mean instrument response of positive control $(n=12) /$ mean instrument response of negative control $(n=12)$

beads, they would not contribute significantly to the change in mass on the membrane and would not be detected.

b) The assay format does not require an off-line wash step. This may help improve the detection of low affinity antibodies.

c) The format utilizes a protein A detection surface. Protein A has five repeated $\mathrm{IgG}$ binding domains which increases assay sensitivity compare to utilization of an anti-IgG as the detector antibody.

In our internal assay development, evaluation of other assay formats and technologies (MSD and AlphaLISA) with PEG.2 as the positive control only achieved sensitivities in the $50-500 \mu \mathrm{g} / \mathrm{mL}$ range (data not shown). Furthermore, experiments on the ViBE (data not shown) using anti-fluorescein on the membrane and FITC-labeled anti-human Fc antibodies as the detection system resulted in high background and apparent non-specific binding that could not be overcome with washes or separation steps which suggested the need for an alternative format.

Some of the key factors to be considered in the development of a sensitive anti-PEG assay are:

\section{Positive Control}

We have found that not all positive controls are detectable across all platforms and assay formats. Hence, it is very important to use well-characterized monoclonal or polyclonal antibodies to try to understand advantages and disadvantages across assay formats and platforms.

2. Assay Buffer

In the development of this assay, multiple commercial and "home-made" blocking buffers were tested. For this particular format, Casein in PBS from Thermo Fisher Scientific provided the lowest signal for the

Table I. Intra- and Inter-Assay Precision Values for Anti-PEG AMMP Assay Reproducibility

\begin{tabular}{|c|c|c|c|c|c|c|c|}
\hline \multirow[b]{2}{*}{ Statistic } & \multicolumn{7}{|c|}{ Nominal concentration $(\mu \mathrm{g} / \mathrm{mL})$} \\
\hline & 40.0 & 20.0 & 5.0 & 1.3 & 0.8 & 0.6 & 0.0 \\
\hline$N$ & 12 & 12 & 12 & 12 & 12 & 12 & 12 \\
\hline Mean AMMP Signal & 0.822 & 0.725 & 0.461 & 0.218 & $0.182 *$ & 0.159 & 0.14 \\
\hline SD & 0.017 & 0.026 & 0.065 & 0.048 & 0.023 & 0.042 & 0.019 \\
\hline Normalized Signal & 5.87 & 5.18 & 3.29 & 1.56 & 1.30 & 1.14 & 1.0 \\
\hline Intrabatch $(\% \mathrm{CV})$ & 0.0 & 0.1 & 0.7 & 1.2 & 1.2 & 1.8 & 1.7 \\
\hline Interbatch $(\% \mathrm{CV})$ & 0.0 & 0.1 & 1.3 & 3.9 & 2.9 & 6.7 & 6.4 \\
\hline
\end{tabular}


NQC and in turn the best assay sensitivity. However, we have observed lot-to-lot variability in assay performance.

3. Labeling Ratio of PEG/mg Bead

During assay development, we found that beads labeled with 30 or $40 \mu \mathrm{g}$ of a PEGylated protein per mg of beads gave lower background signal compared to the beads labeled with 10 or $20 \mu \mathrm{g}$ of protein per $\mathrm{mg}$ of bead in buffers containing $5 \%$ human serum. This finding suggested that higher protein coverage of the bead surface helped decrease non-specific binding of other serum proteins to the beads. However, when beads were labeled with PEG alone (no protein), $20 \mu \mathrm{g}$ of PEG per mg beads had lower background and better assay sensitivity than beads labeled with $30 \mu \mathrm{g}$. In the future, studies around the orientation of the PEG on the beads may help better understand the results.

4. Removal of Tween-20 or Any Other Surfactants that Resemble PEG from the Assay Buffer

Sherman et al. $(14,15)$ previously reported on the detrimental effects of such surfactants on the sensitivity of detection for anti-PEG antibodies. Since our positive control antibody is PEG backbone unit specific, the interference was expected. In our internal assay development, we also observed that Tween-20 in the wash buffer reduced assay sensitivity. In a direct MSD buffer assay with a polyclonal antibody as positive control, nice dose-response curves were observed with three different PEGs tested (0.35, 0.75 , and $40 \mathrm{kDa}$ ) when only PBS was used as wash buffer. The dose responding curves disappeared when $0.05 \%$ Tween 20 was added to PBS wash buffer. We are currently exploring other detergents which do not have structural resemblance to PEG.

This manuscript is the first report in which the ViBE platform is used to detect anti-PEG antibodies and the first report on adequate sensitivity for detection of an $\mathrm{IgG}$ anti-PEG control. As a rapid communication, it only touches on some of the key aspects to the development of an anti-PEG assay. We have recently undertaken the task of comparing a several anti-PEG positive controls from our inventory across at least two platforms to deepen our understanding of advantages and potential pitfalls of each.

\section{CONCLUSION}

An Acoustic Membrane MicroParticle method for detection of anti-PEG antibodies using the ViBE instrument from BioScale has been found to be reproducible $(\% \mathrm{CV}<7)$ and with a sensitivity of near $1000 \mathrm{ng} / \mathrm{mL}$ in human serum samples. This preliminary work is intended to assist researchers developing assays for detection of anti-PEG antibodies and will be expanded upon.

\section{ACKNOWLEDGMENTS}

The authors would like to acknowledge the work done by Murli Krishna and Holly Palmé in the oversight of reagent generation for this program; Steven Piccoli for review of this manuscript.

\section{REFERENCES}

1. Gorovits B, Wakshull E, Pillutla R, Xu Y, Starcevic M, Goyal J. Recommendations for the characterization of immunogenicity response to multiple domain biotherapeutics. J Immunol Methods. 2014;408:1-12.

2. Guidance for Industry Immunogenicity Assessment for Therapeutic Proteins, Sept 2014. Available from:http://www.fda.gov/ downloads/Drugs/GuidanceComplianceRegulatoryInformation/ Guidances/UCM338856.pdf

3. Schellekens H, Hennink WE, Brinks V. The immunogenicity of polyethylene glycol: facts and fiction. Pharm Res. 2013;30(7):1729-34.

4. Hershfield MS, Ganson NJ, Kelly SJ, Scarlett EL, Jaggers DA, Sundy JS. Induced and pre-existing anti-polyethylene glycol antibody in a trial of every 3-week dosing of pegloticase for refractory gout, including in organ transplant recipients. Arthritis Res Ther. 2014;16:R63.

5. Myler H, Hruska MW, Srinivasan S, Cooney E, Kong G, Dodge $\mathrm{R}$, et al. PEG immunogenicity: a clinical case study with peginterferon lambda $1-a$ and peginterferon Alfa-1 $2 a$ in naive patients. Bioanalysis. 2015;7(9):1093-106.

6. Liu Y, Reidler H, Pan J, Milunic D, Qin D, Chen D, et al. A double antigen bridging immunogenicity ELISE for the detection of antibodies to polyethylene glycol polymers. J Pharmacol Toxicol Methods. 2011;64(3):245-56.

7. Wiberg M, Wang D, Yang L, Kamerud J. Detection of human anti-polyethyene glycol (PEG) antibodies: challenges for method development. http://www.aapsj.org/abstracts/AM_2010/ T3292.pdf

8. Ishida T, Wang X, Shimizu T, Nawata K, Kiwada H. PEGylated liposome elicit anti-PEG IgM response in a T cell-independent manner. J Control Release. 2007;122(3):349-55.

9. Ichihara M, Shimizu T, Imoto A, Hashiguchi Y, Uehara Y, Ishida T. Anti-PEG IgM response against PEGylated liposomes in mice and rats. Pharmaceutics. 2011;3:1-11.

10. Chilewski SD, Dickerson WM, Mora JR, Saab A, Alderman EM. Evaluation of acoustic membrane microparticle (AMMP) technology for a sensitive ligand binding assay to support pharmacokinetic determinations of a biotherapeutic. AAPS J. 2014;16(6):1366-71.

11. Krishna M, Palme H, Duo J, Lin Z, Corbett M, Dodge R. Development and characterization of antibody reagents to assess anti PEG IgG antibodies in clinical samples. Bioanalysis. 2015.

12. Garay RP, EL-Gewely R, Armstrong JK, Garratty G, Richette P. Antibodies against polyethyene glycol in health subject and in patients treated with PEG-conjugated agents. Expert Opin Drug Deliv. 2012;9(11):1319-23.

13. Ishida T, Kiwada H. Anti-polyethyleneglycol antibody response to PEGylated substances. Biol Pharm Bull. 2013;36(6):889-91.

14. Sherman MR, Williams LD, Sobczyk MA, Michaels SJ, Saifer MG. Role of the methoxy group in immune responses to mPEGprotein conjugates. Bioconjug Chem. 2012;23:485-99.

15. Saifer MG, Williams LD, Sobczyk MA, Michaels SJ, Sherman MR. Selectivity of binding of PEGs and PEG-like oligomers to anti-PEG antibodies induced by methoxyPEG-proteins. Mol Immunol. 2014;57(2):236-46. 